\title{
HYDROCEPHALUS AFTER INTRA-UTERINE FUNGAL INFECTION
}

\author{
BY \\ A. F. BURRY \\ From the Christchurch Hospital, New Zealand
}

(RECEIVED FOR PUBLICATION OCTOBER 30, 1956)

Fungal infections of the nervous system are uncommon but are mentioned by Russell (1949) as a possible cause of hydrocephalus. Intra-uterine meningitis from any cause has been reported only once. Crosby, Mosberg and Smith (1951), in reporting this case, reviewed the literature on meningitis of the newborn up to 1951 and could find no other case acceptable as having an

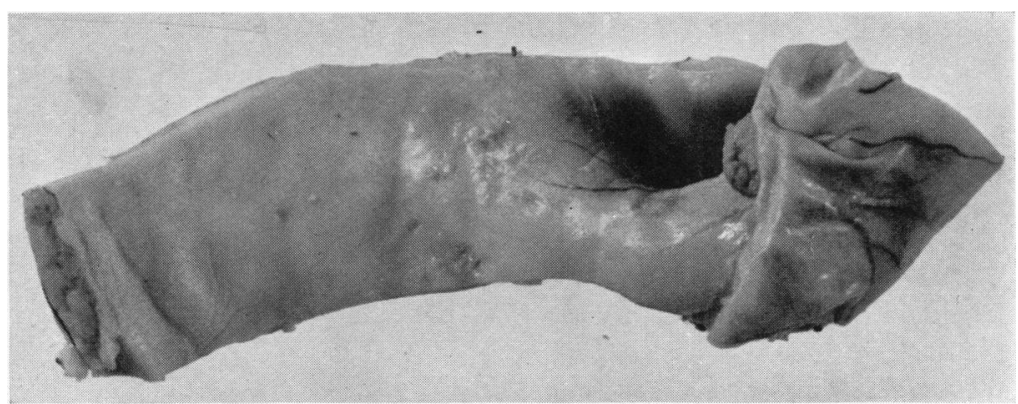

Fig. 2.-Lining of lateral ventricle showing roughening and granules. inception in the intra-uterine period.

The following case appears to be unique in that hydrocephalus resulted from an intra-uterine attack of meningitis due to a fungus.

\section{Case Report}

Baby S. was born on August 8, 1954, after a short, forceful labour lasting two hours. The presentation was vertex, L.O.A. The mother's health had been normal throughout the pregnancy. There was no history suggestive of vaginal thrush on close questioning, and antibiotics had not been given during the pregnancy. The only previous child, born in 1949, was alive and well.

Conception in the present case had followed closely

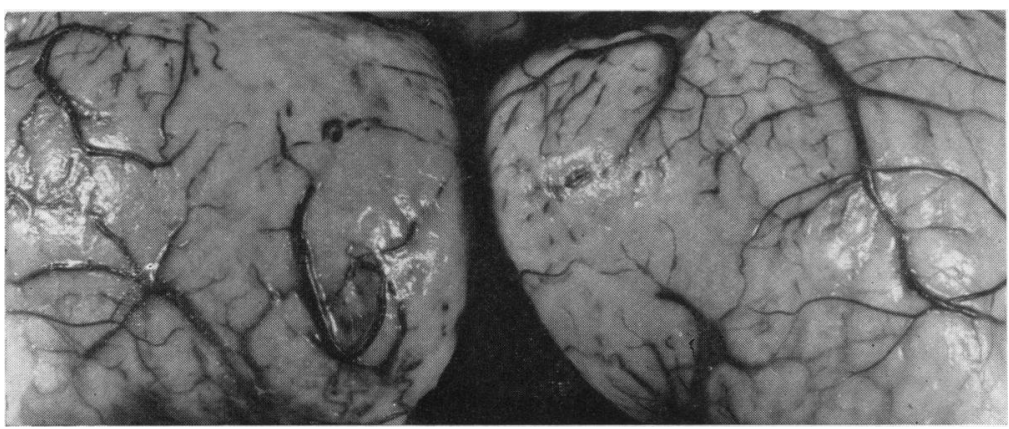

FIG. 1.-Occipital poles showing organized exudate in subarachnoid space. on dilatation and curettage and tubal insufflation, carried out because of apparent infertility.

The baby, a boy, weighed $7 \frac{1}{2} \mathrm{lb}$. at birth. He was slow to breathe and cry but responded well to oxygen and incubation. At the time of delivery some haemorrhagic areas were noted on the face, attributed to bruising at delivery. These faded within a fortnight. Soon after birth it was noticed that the baby's sucking and swallowing reflexes were poor and for the first fortnight he was tube fed. For the first $\mathbf{1 0}$ days his tenure of life was precarious. Twitching of the left eyelid was first noted on the third day and subsequently both eyes and the left side of the face twitched at intervals. Examination on the fifth day showed undue separation of the skull sutures and a diagnosis of hydrocephalus was suggested.

From the end of the second week there was some improvement in the baby's condition. Sucking improved and bottle feeding was started. The head diminished in size from a circumference of $15 \frac{1}{2}$ in. on the 18 th day to $14 \frac{1}{2}$ in. on the 29th day. On the 33rd day he had a convulsion and began to pass frequent green motions. His condition progressively deteriorated and he died on the 37th day.

Post-mortem examination was performed by the author 18 hours after death. The body was that of a normally developed but 

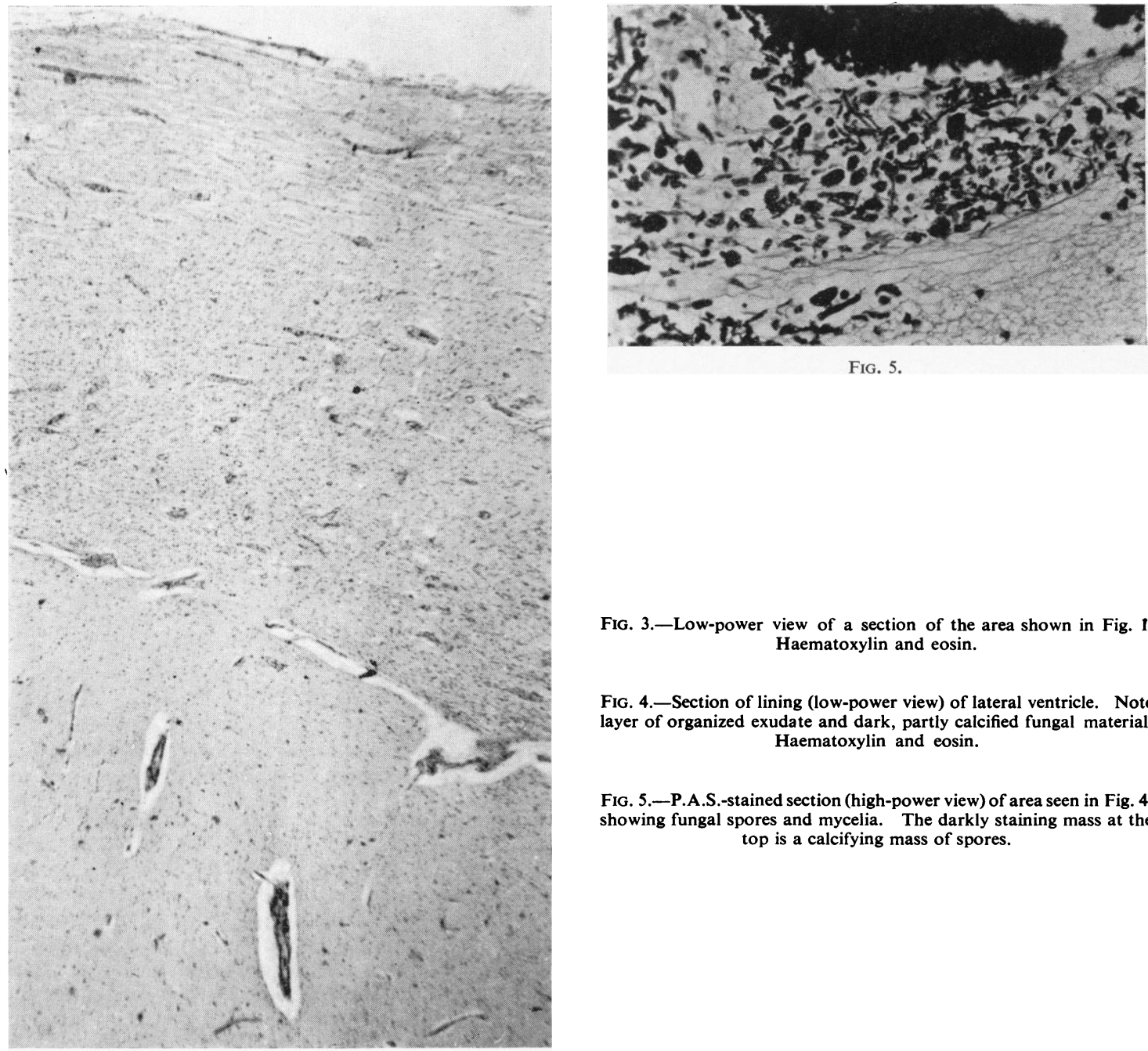

FIG. 5.

Fig. 3.-Low-power view of a section of the area shown in Fig. 1. Haematoxylin and eosin.

Fig. 4.-Section of lining (low-power view) of lateral ventricle. Note layer of organized exudate and dark, partly calcified fungal material Haematoxylin and eosin.

FIG. 5.-P.A.S.-stained section (high-power view) of area seen in Fig. 4, showing fungal spores and mycelia. The darkly staining mass at the top is a calcifying mass of spores.

FIG. 3.

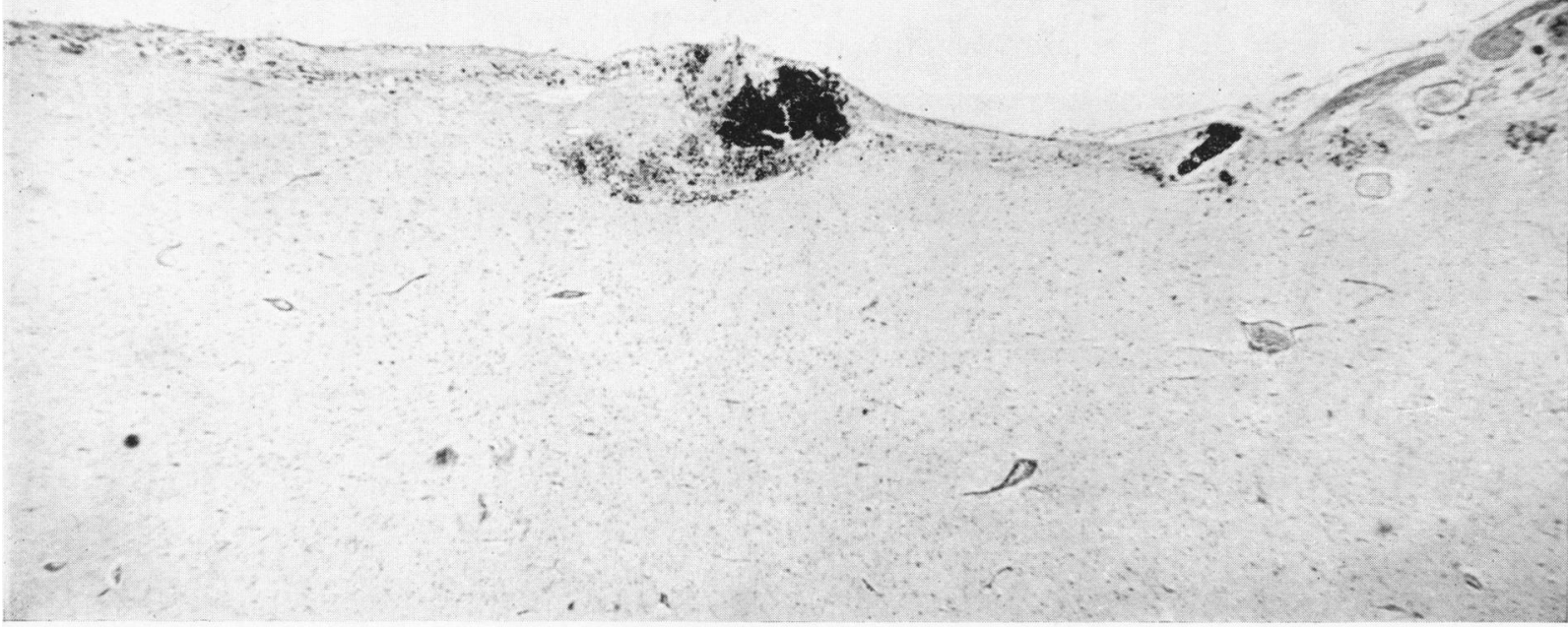


rather poorly nourished male infant weighing $3,280 \mathrm{~g}$. The trachea and bronchi contained yellowish mucoid material and the lungs showed basal collapse and congestion. The small intestine was slightly dilated and contained watery yellowish fluid and the contents of the large bowel were also watery and yellow.

The skull measured 14 in. in circumference, the cranial bones were thinned and the sutures were widened. The cerebral hemispheres were tense and distended with marked flattening of the sulci. A striking feature was the obliteration of sulcal markings over the occipital poles by pale organized exudate (Fig. 1). There were a few fine adhesions about the base of the brain. There was gross hydrocephalus involving the lateral and third ventricles. The corpus callosum was grossly thinned. The aqueduct was narrow but patent. The lining of the lateral ventricles was roughened at some points and there were scattered small yellowish projections into the lumen (Fig. 2).

The possibility of toxoplasmosis was entertained and the eyes were examined and sectioned but were normal macroscopically and microscopically.

Sections of the exudate over the occipital pole and to a lesser extent at other points, mainly at the base of the brain, showed marked meningeal thickening with adhesions to the pia (Fig. 3).

No organisms could be demonstrated in the organized exudate which showed very sparse lymphocytic infiltration. Sections from the lining of the lateral ventricles showed partial loss of the ependymal layer, the cells of which were elsewhere largely flattened to cuboidal. Beneath a layer of glial scarring, and at some points penetrating more deeply into the brain substance, were irregular masses of partly calcified necrotic material and structures identifiable by P.A.S. stains as hyphae and spores of a fungus (Figs. 4, 5). These masses of fungi and necrotic material formed the yellowish granules visible macroscopically. There was a sparse lymphocytic infiltration around the fungal masses.

Sections from a number of other organs, including lung, liver and pancreas, showed no evidence of disseminated fungal infection.

The final morbid anatomical diagnosis was hydrocephalus after intra-uterine fungal infection, gastroenteritis and inhalation of vomitus.

\section{Comment}

Was the infection truly intra-uterine? There are several reasons for this belief. First the distribution of exudate over the occipital pole is best explained by the baby presenting as a vertex in the L.O.A. position when the occipital poles would be dependent. Secondly the histological appearances with well established scarring and calcification suggest a longer duration of the inflammatory process than five weeks. Thirdly the baby was feeble from birth and hydrocephalus was suspected from as early as the fifth day. It is hardly likely that hydrocephalus could develop in such a short time if a post-natal infection were postulated.

Source of Infection. Monilial vaginal infections are common during pregnancy and it is possible that this patient had a subclinical infection which in some way reached the placenta and was transmitted through it. Another possibility is that the infection was introduced to tubes or uterine cavity in the course of the dilatation and curettage and tubal insufflation carried out not long before conception took place. The infecting organism passing through the placenta may have reached the choroidal plexus and primarily infected the lateral ventricles. The small calibre of the aqueduct could have diminished the spread of infection to the meninges, or the absence of demonstrable organisms in the meninges might be due to the more efficient response of the meninges to infection.

Nature of the Infection. Unfortunately no cultures were made before fixation of the brain so that identification of the infecting organism is incomplete. The presence of septate pseudomycelia and small spores suggests that Candida albicans, the most likely pathogen, is responsible for this infection.

Cause of the Hydrocephalus. The clinical course with apparent diminution in the degree of hydrocephalus suggests that a blockage in the aqueduct may have resolved. Morris, Kalz and Lotspeich (1945), describing a case of monilial meningitis, similarly noted that the reactive process was greater in the ventricular cavities than in the subarachnoid space.

\section{Summary}

A case is described of hydrocephalus in an infant, due to a fungal infection of the meninges. Reasons are given for the belief that the infection was intrauterine.

I have to thank Dr. L. C. Averill for permission to publish this case and Drs. D. T. Stewart and G. C. T. Burns and Professor J. A. R. Miles for their help and advice.

REFERENCES

Crosby, R. M. N., Mosberg, W. H. and Smith, G. W. (1951). J. Pediat., 39, 94.

Morris, A. A., Kalz, G. G. and Lotspeich, E. S. (1945). Arch. Neurol. Psychiat. (Chicago)), 54, 361.

Russell, D. S. (1949). Spec. Rep. Ser. med. Res. Coun. (Lond.). No. 265 . 